\title{
LACUNARY DIFFERENCE SEQUENCES OF COMPLEX UNCERTAIN VARIABLES
}

\author{
PRANAB JYOTI DOWARI AND BINOD CHANDRA TRIPATHY
}

\begin{abstract}
Using the concept of difference operator on sequence spaces and uncertainty theory, some new class of lacunary convergent difference sequences of complex uncertain variables have been introduced for the lacunary convergence. Some topological properties of the defined sequence spaces along with the inclusion relations have been investigated.
\end{abstract}

Користуючись поняттям різницевого оператора на просторі послідовностей і теорією невизначеності, ми досліджуємо клас лакунарно збіжних різницевих послідовностей комплексних невизначених змінних. Розглядаються топологічні властивості введених просторів послідовностей, а також їхні вкладення.

\section{INTRODUCTION}

The concept of uncertainty theory was introduced by Liu 9] in the year 2007. Nowa-days uncertainty theory has become a thrust area of research in various branches of mathematics such as uncertain programming, uncertain risk analysis, uncertain logic,etc. In our daily life there exists uncertainty such as randomness and fuzziness. Probability theory is used to model frequencies of random events whereas uncertainty theory is used to model belief degree of an event to be true. The uncertainty theory is based on uncertain measure which satisfies normality, duality, subadditivity and product axioms. Complex uncertain variables are measurable functions from uncertainty spaces to the set of complex numbers. Convergence of sequences always plays a crucial role in different theory of mathematics. Chen et al. [19] first introduced the convergence concepts of complex uncertain variables. Further study on complex uncertain variables are done by Tripathy and Nath [6], Tripathy and Dowari [7, Kisi [15,16], Dowari and Tripathy [1], You [10] and many others.

The initial works on lacunary sequences was done by Freedman et al. 2. Their studies of the $\left|\sigma_{1}\right|$ of strongly Cesàro summable sequences with general lacunary $\theta$ resulted in introducing a larger class of sequences $N_{\theta}$ called lacunary sequences. Later their works have been further extended by many researchers. The main concern of this current study is to extend the classes with the uncertain sequences in the uncertainty space and the lacunary convergence concepts of complex uncertain sequences with respect to difference sequence spaces.

\section{Preliminaries}

Let $\omega$ be the set of all sequences of real or complex numbers and $\ell_{\infty}, c$ and $c_{0}$ be, respectively, the Banach spaces of bounded, convergent and null sequences $x=\left(x_{k}\right)$ with the usual norm $\|x\|=\sup _{k}\left|x_{k}\right|$.

By a lacunary sequence $\theta=\left(k_{r}\right)$; where $k_{0}=0$, we shall mean an increasing sequence of non-negative integers with $k_{r}-k_{r-1} \rightarrow \infty$, as $r \rightarrow \infty$. The intervals determined by $\theta$ will be denoted by $I_{r}=\left(k_{r-1}, k_{r}\right]$ and $q_{r}=\frac{k_{r}}{k_{r-1}}, h_{r}=k_{r}-k_{r-1}$ for $r=1,2,3, \ldots$

2020 Mathematics Subject Classification. 40A05; 40A25; 60B10; 60B12; 60F17.

Keywords. Almost convergence sequences; complex uncertain variable; difference sequence; Lacunary sequence. 
Freedman et al. 2] studied the space of lacunary strongly convergent sequences $N_{\theta}$ and was defined as follows:

$$
N_{\theta}=\left\{x=\left(x_{k}\right): \lim _{r \rightarrow \infty} \frac{1}{h_{r}} \sum_{k \in I_{r}}\left|x_{k}-L\right|=0, \text { for some } L\right\} .
$$

The notion of difference sequence was introduced by Kizmaz [12]. Then Esi, Tripathy and Sarma [1] introduced the generalized difference sequence spaces as follows:

Let $m, n \geq 0$ be fixed integers,

$$
Z\left(\triangle_{m}^{n}\right)=\left\{x=\left(x_{k}\right) \in \omega: \triangle_{m}^{n} x=\left(\triangle_{m}^{n} x_{k}\right) \in Z\right\},
$$

for $Z=\ell_{\infty}, c$ and $c_{0}$; where $\triangle_{m}^{n} x_{k}=\left(\triangle_{m}^{n-1} x_{k}-\triangle_{m}^{n-1} x_{k+m}\right)$ and $\triangle_{m}^{0} x_{k}=x_{k}$ for all $k \in \mathbb{N}$. This generalized difference notion has the following binomial representation:

$$
\triangle_{m}^{n} x_{k}=\sum_{v=0}^{n}(-1)^{v}\left(\begin{array}{l}
n \\
v
\end{array}\right) x_{k+m v} \quad \text { for all } \quad k \in \mathbb{N} .
$$

For $m=1$ and $n=1$, these spaces represent the spaces $\ell_{\infty}(\triangle), c(\triangle)$ and $c_{0}(\triangle)$ introduced and studied by Kizmaz [12]. For $m=1$, these spaces represent the spaces $\ell_{\infty}\left(\triangle^{n}\right), c\left(\triangle^{n}\right)$ and $c_{0}\left(\triangle^{n}\right)$ introduced and studied by Et and Colak [14]. For $n=1$, these spaces represent the spaces $\ell_{\infty}\left(\triangle_{m}\right), c\left(\triangle_{m}\right)$ and $c_{0}\left(\triangle_{m}\right)$ introduced and studied by Tripathy and Esi [5].

The sequence spaces $Z\left(\triangle_{m}^{n}\right)$ for $Z=\ell_{\infty}, c$ and $c_{0}$ are Banach spaces, by the norm

$$
\|x\|_{\triangle_{m}^{n}}=\sum_{i=1}^{p}\left|x_{i}\right|+\sup _{k}\left|\triangle_{m}^{n} x_{k}\right|
$$

where $p=m n$ for $m \geq 1, n \geq 1$.

Now, we discuss briefly about the uncertainty theory which is introduced in [9].

Let $\mathcal{L}$ be a $\sigma$-algebra on a nonempty set $\Gamma$. A set function $\mathcal{M}$ is called an uncertain measure if it satisfies the following axioms:

Axiom 1 (Normality Axiom). $\mathcal{M}\{\Gamma\}=1$;

Axiom 2 (Duality Axiom). $\mathcal{M}\{\Lambda\}+\mathcal{M}\left\{\Lambda^{c}\right\}=1$ for any $\Lambda \in \mathcal{L}$;

Axiom 3 (Subadditivity Axiom). For every countable sequence of $\left\{\lambda_{j}\right\} \in \mathcal{L}$, we have

$$
\mathcal{M}\left\{\bigcup_{j=1}^{\infty} \lambda_{j}\right\} \leq \sum_{j=1}^{\infty} \mathcal{M}\left\{\lambda_{j}\right\} .
$$

The triplet $(\Gamma, \mathcal{L}, \mathcal{M})$ is called an uncertainty space, and each element $\Lambda$ in $\mathcal{L}$ is called an event. In order to obtain an uncertain measure of compound event, a product uncertain measure is defined by Liu [9] as follows:

Axiom 4 (Product Axiom). Let $\left(\Gamma_{k}, \mathcal{L}_{k}, \mathcal{M}_{k}\right)$ be uncertainty space for $k=1,2,3, \ldots$ The product uncertain measure $\mathcal{M}$ is an measure satisfying

$$
\mathcal{M}\left\{\prod_{k=1}^{\infty} \Lambda_{k}\right\}=\bigwedge_{k=1}^{\infty} \mathcal{M}_{k}\left\{\Lambda_{k}\right\}
$$

where $\Lambda_{k}$ are arbitrarily chosen events from $\mathcal{L}_{k}$ for $k=1,2, \ldots$, respectively.

A complex uncertain variable is a measurable function $\xi$ from an uncertainty space $(\Gamma, \mathcal{L}, \mathcal{M})$ to the set of complex numbers, i.e., for any Borel set $\mathcal{B}$ of complex numbers, the set

$$
\{\xi \in \mathcal{B}\}=\{\gamma \in \Gamma: \xi(\gamma) \in \mathcal{B}\}
$$


is an event. When the range is the set of real numbers, we call it as an uncertain variable, introduced and investigated by Liu[9]. As a complex function on uncertainty space, complex uncertain variable is mainly used to model a complex uncertain quantity. The expected value operator of an uncertain variable was defined by Liu[9] as

$$
E[\xi]=\int_{0}^{+\infty} \mathcal{M}\{\xi \geq r\} d r-\int_{-\infty}^{0} \mathcal{M}\{\xi \leq r\} d r
$$

provided that at least one of the two integrals is finite.

The complex uncertainty distribution $\Phi(x)$ of a complex uncertain variable $\xi$ is a function from $\mathbb{C}$ to $[0,1]$ defined by

$$
\Phi(c)=\mathcal{M}\{\operatorname{Re}(\xi) \leq \operatorname{Re}(c), \operatorname{Im}(\xi) \leq \operatorname{Im}(c)\}
$$

for any complex number $c$.

An uncertain variable is said to be positive, when it maps from $\mathbb{R}_{+} \bigcup\{0\}$ (non-negative real numbers) to $[0,1]$. Considering the important role of sequence convergence in mathematics, some concepts of convergence for complex uncertain sequences were introduced in Chen, Ning, Wang [19]. Complex uncertain sequences are sequence of complex uncertain variables indexed by integers.

The complex uncertain sequence $\left\{\xi_{n}\right\}$ is said to be convergent almost surely(a.s.) to $L$ if there exists an event $\Lambda$ with $\mathcal{M}\{\Lambda\}=1$ such that

$$
\lim _{n \rightarrow \infty}\left\|\xi_{n}(\gamma)-L(\gamma)\right\|=0
$$

for every $\gamma \in \Lambda$. In that case we write $\xi_{n} \rightarrow L$, a.s..

The complex uncertain sequence $\left\{\xi_{n}\right\}$ is said to be convergent in measure to $L$ if for a given $\varepsilon>0$,

$$
\lim _{n \rightarrow \infty} \mathcal{M}\left\{\left\|\xi_{n}(\gamma)-L(\gamma)\right\| \geq \varepsilon\right\}=0
$$

The complex uncertain sequence $\left\{\xi_{n}\right\}$ is said to be convergent in mean to $L$ if

$$
\lim _{n \rightarrow \infty} E\left[\left\|\xi_{n}(\gamma)-L(\gamma)\right\|\right]=0 .
$$

Let $\Phi, \Phi_{1}, \Phi_{2}, \Phi_{3}, \ldots$ be the complex uncertainty distributions of complex uncertain variables $\xi, \xi_{1}, \xi_{2}, \xi_{3} \ldots$, respectively. We say the complex uncertain sequence $\left\{\xi_{n}\right\}$ converges in distribution to $L$ if

$$
\lim _{n \rightarrow \infty} \Phi_{n}(c)=\Phi(c)
$$

for all $c \in \mathbb{C}$, at which $\Phi(c)$ is continuous.

The complex uncertain sequence $\left\{\xi_{n}\right\}$ is said to be convergent uniformly almost surely(u.a.s.) to $L$ if there exists a sequence of events $\left\{E_{k}^{\prime}\right\}, \mathcal{M}\left\{E_{k}^{\prime}\right\} \rightarrow 0$ such that $\left\{\xi_{n}\right\}$ converges uniformly to $L$ in $\Gamma-E_{k}^{\prime}$, for any fixed $k \in \mathbb{N}$.

\section{Definitions and Results}

In this section we define some new classes of sequences of uncertain variables using the concept of difference operator on sequences.

$\left|\sigma_{1}\right|^{U}\left(\triangle_{m}^{n}\right)=\left\{\xi=\left(\xi_{i}(\gamma)\right)\right.$ : there exists $L(\gamma)$ such that $\frac{1}{n} \sum_{i=1}^{n}\left\|\triangle_{m}^{n} \xi_{i}(\gamma)-L(\gamma)\right\| \rightarrow 0$, as $n \rightarrow \infty\}$.

Let $\theta=\left(k_{r}\right)$ be a lacunary sequence and $\left(\xi_{k}\right)$ be a sequence of uncertain variables in the space $(\Gamma, \mathcal{L}, \mathcal{M})$. 
We define the following classes of sequences:

$$
\begin{aligned}
& {\left[N_{\theta}^{U}, \triangle_{m}^{n}\right]_{0} }=\left\{\xi=\left(\xi_{k}\right): \lim _{r \rightarrow \infty} \frac{1}{h_{r}} \sum_{k \in I_{r}}\left\|\triangle_{m}^{n} \xi_{k}(\gamma)\right\|=0\right\} \\
& {\left[N_{\theta}^{U}, \triangle_{m}^{n}\right]_{1}=\left\{\xi=\left(\xi_{k}\right): \lim _{r \rightarrow \infty} \frac{1}{h_{r}} \sum_{k \in I_{r}}\left\|\triangle_{m}^{n} \xi_{k}(\gamma)-L(\gamma)\right\|=0\right.}\text { for some } L(\gamma) \in(\Gamma, \mathcal{L}, \mathcal{M})\}, \\
& {\left[N_{\theta}^{U}, \triangle_{m}^{n}\right]_{\infty}=\left\{\xi=\left(\xi_{k}\right): \sup _{r} \frac{1}{h_{r}} \sum_{k \in I_{r}}\left\|\triangle_{m}^{n} \xi_{k}(\gamma)\right\|<\infty\right\}, } \\
& {\left[\left|A C_{\theta}\right|^{U}, \triangle_{m}^{n}\right]=\left\{\xi=\left(\xi_{k}\right): \lim _{r \rightarrow \infty} \frac{1}{h_{r}} \sum_{k \in I_{r}}\left\|\triangle_{m}^{n} \xi_{k+n}(\gamma)-L(\gamma)\right\|=0, \text { uniformly in } n\right\} . }
\end{aligned}
$$

A sequence space $E$ is said to be solid (or normal) if $\left(\alpha_{k} x_{k}\right) \in E$, whenever $\left(x_{k}\right) \in E$, for all sequences $\left(\alpha_{k}\right)$ of scalars such that $\left|\alpha_{k}\right| \leq 1$ for all $k \in \mathbb{N}$. The sequence space is said to be symmetric if $\left(x_{k}\right) \in E \Rightarrow\left(x_{\pi(k)}\right) \in \lambda$, where $\pi$ is a permutation of $\mathbb{N}$. The sequence space $E$ is said to be monotone if $E$ contains the canonical pre-images of all its step spaces.

Lemma 3.1. If a sequence space is solid then it is monotone.

With the new concepts defined above we now consider the following results.

Theorem 3.1. The classes of complex uncertain sequences $\left[N_{\theta}^{U}, \triangle_{m}^{n}\right]_{0},\left[N_{\theta}^{U}, \triangle_{m}^{n}\right]_{1}$ and $\left[N_{\theta}^{U}, \triangle_{m}^{n}\right]_{\infty}$ are linear spaces.

Proof. We establish the result for the class of complex uncertain sequence $\left[N_{\theta}^{U}, \triangle_{m}^{n}\right]_{0}$. The other cases will follow similarly. Let $\left(\xi_{k}\right),\left(\eta_{k}\right) \in\left[N_{\theta}^{U}, \triangle_{m}^{n}\right]_{0}$. Then we have,

$$
\begin{gathered}
\lim _{r \rightarrow \infty} \frac{1}{h_{r}} \sum_{k \in I_{r}}\left\|\triangle_{m}^{n} \xi_{k}(\gamma)\right\|=0 \\
\text { and } \lim _{r \rightarrow \infty} \frac{1}{h_{r}} \sum_{k \in I_{r}}\left\|\triangle_{m}^{n} \eta_{k}(\gamma)\right\|=0 .
\end{gathered}
$$

Now for $\alpha, \beta \in \mathbb{C}$,

$$
\begin{aligned}
& \left.\lim _{r \rightarrow \infty} \frac{1}{h_{r}} \sum_{k \in I_{r}}\left\|\triangle_{m}^{n}\left(\alpha \xi_{k}(\gamma)+\beta \eta_{k}(\gamma)\right)\right\|=\lim _{r \rightarrow \infty} \frac{1}{h_{r}} \sum_{k \in I_{r}} \| \alpha \triangle_{m}^{n} \xi_{k}(\gamma)+\beta \triangle_{m}^{n} \eta_{k}(\gamma)\right) \| \\
& \left.\quad \leq|\alpha| \lim _{r \rightarrow \infty} \frac{1}{h_{r}} \sum_{k \in I_{r}}\left\|\triangle_{m}^{n} \xi_{k}(\gamma)+|\beta| \lim _{r \rightarrow \infty} \frac{1}{h_{r}} \sum_{k \in I_{r}}\right\| \triangle_{m}^{n} \eta_{k}(\gamma)\right) \| \rightarrow 0, \quad \text { as } \quad r \rightarrow \infty .
\end{aligned}
$$

Hence $\left(\alpha \xi_{k}(\gamma)+\beta \eta_{k}(\gamma)\right) \in\left[N_{\theta}^{U}, \triangle_{m}^{n}\right]_{0}$. Therefore $\left[N_{\theta}^{U}, \triangle_{m}^{n}\right]_{0}$ is a linear space.

Theorem 3.2. The classes of complex uncertain sequences $\left[N_{\theta}^{U}, \triangle_{m}^{n}\right]_{0},\left[N_{\theta}^{U}, \triangle_{m}^{n}\right]_{1}$ and $\left[N_{\theta}^{U}, \triangle_{m}^{n}\right]_{\infty}$ are normed linear spaces, normed by

$$
\|\xi(\gamma)\|_{\triangle_{m}^{n}}=\sum_{i=1}^{p}\left\|\xi_{i}(\gamma)\right\|+\sup _{r} \frac{1}{h_{r}} \sum_{k \in I_{r}}\left\|\triangle_{m}^{n} \xi_{k}(\gamma)\right\|
$$

where $p=m n$ for $m \geq 1, n \geq 1$.

Proof. For $\xi=\theta$, we have $\|\theta\|_{\triangle_{m}^{n}}=0$.

Conversely let $\|\xi(\gamma)\|_{\triangle_{m}^{n}}=0$. 
Then $\|\xi(\gamma)\|_{\triangle_{m}^{n}}=\sum_{i=1}^{p}\left\|\xi_{i}(\gamma)\right\|+\sup _{r} \frac{1}{h_{r}} \sum_{k \in I_{r}}\left\|\triangle_{m}^{n} \xi_{k}(\gamma)\right\|=0$

$\Rightarrow \xi_{i}(\gamma)=0$, for $i=1,2, \ldots, m n$ and $\left\|\triangle_{m}^{n} \xi_{k}(\gamma)\right\|=0$ for $k \in I_{r}, r=1,2, \ldots$

Consider $k=1$, i.e., $\left\|\triangle_{m}^{n} \xi_{1}(\gamma)\right\|=0 \Rightarrow\left\|\triangle_{m}^{n-1} \xi_{1}(\gamma)-\triangle_{m}^{n-1} \xi_{m+1}(\gamma)\right\|=0 \Rightarrow$ $\xi_{m+1}(\gamma)=0$, since $\xi_{i}(\gamma)=0$, for $i=1,2, \ldots, m n$

Proceeding inductively we can have $\xi_{k}(\gamma)=0$, for all $k \in I_{r}$.

$$
\begin{array}{r}
\|\xi(\gamma)+\eta(\gamma)\|_{\triangle_{m}^{n}}=\sum_{i=1}^{p}\left\|\xi_{i}(\gamma)+\eta_{i}(\gamma)\right\|+\sup _{r} \frac{1}{h_{r}} \sum_{k \in I_{r}}\left\|\triangle_{m}^{n}\left(\xi_{k}(\gamma)+\eta_{k}(\gamma)\right)\right\| \\
\leq \sum_{i=1}^{p}\left\|\xi_{i}(\gamma)\right\|+\sup _{r} \frac{1}{h_{r}} \sum_{k \in I_{r}}\left\|\triangle_{m}^{n} \xi_{k}(\gamma)\right\|+\sum_{i=1}^{p}\left\|\eta_{i}(\gamma)\right\|+\sup _{r} \frac{1}{h_{r}} \sum_{k \in I_{r}}\left\|\triangle_{m}^{n} \eta_{k}(\gamma)\right\| \\
=\|\xi(\gamma)\| \triangle_{m}^{n}+\|\eta(\gamma)\|_{\triangle_{m}^{n}} .
\end{array}
$$

Now, for any $\lambda \in \mathbb{C}$,

$$
\begin{aligned}
\|\lambda \xi(\gamma)\|_{\triangle_{m}^{n}} & =\sum_{i=1}^{p}\left\|\lambda \xi_{i}(\gamma)\right\|+\sup _{r} \frac{1}{h_{r}} \sum_{k \in I_{r}}\left\|\triangle_{m}^{n}\left(\lambda \xi_{k}(\gamma)\right)\right\| \\
& =|\lambda|\|\xi(\gamma)\|_{\triangle_{m}^{n}}
\end{aligned}
$$

This completes the proof.

Theorem 3.3. For $m \geq 1$ and $n \geq 1 \quad\left[N_{\theta}^{U}, \triangle_{m}^{n-1}\right]_{z} \subset\left[N_{\theta}^{U}, \triangle_{m}^{n}\right]_{z}$ for $z=0,1, \infty$. In general, $\left[N_{\theta}^{U}, \triangle_{m}^{i}\right]_{z} \subset\left[N_{\theta}^{U}, \triangle_{m}^{n}\right]_{z}$ for $z=0,1, \infty$ and $i=0,1, \ldots, n-1$. The inclusions are strict.

Proof. Let $\left\{\xi_{k}\right\} \in\left[N_{\theta}^{U}, \triangle_{m}^{n-1}\right]_{0}$.

Then we have,

$$
\lim _{r \rightarrow \infty} \frac{1}{h_{r}} \sum_{k \in I_{r}}\left\|\triangle_{m}^{n-1} \xi_{k}(\gamma)\right\|=0
$$

Now,

$$
\begin{aligned}
\frac{1}{h_{r}} \sum_{k \in I_{r}}\left\|\triangle_{m}^{n} \xi_{k}(\gamma)\right\| & =\frac{1}{h_{r}} \sum_{k \in I_{r}}\left\|\triangle_{m}^{n-1} \xi_{k}(\gamma)-\triangle_{m}^{n-1} \xi_{k+1}(\gamma)\right\| \\
& \leq\left(\frac{1}{h_{r}} \sum_{k \in I_{r}}\left\|\triangle_{m}^{n-1} \xi_{k}(\gamma)\right\|-\frac{1}{h_{r}} \sum_{k \in I_{r}}\left\|\triangle_{m}^{n-1} \xi_{k+1}(\gamma)\right\|\right)
\end{aligned}
$$

Taking limit as $r \rightarrow \infty$ we have,

$$
\frac{1}{h_{r}} \sum_{k \in I_{r}}\left\|\triangle_{m}^{n} \xi_{k}(\gamma)\right\|=0, \quad \operatorname{by}(3.1)
$$

which implies $\left\{\xi_{k}\right\} \in\left[N_{\theta}^{U}, \triangle_{m}^{n}\right]_{0}$.

The other cases will follow similarly.

Proceeding inductively we have, $\left[N_{\theta}^{U}, \triangle_{m}^{i}\right]_{z} \subset\left[N_{\theta}^{U}, \triangle_{m}^{n}\right]_{z} \infty$ and $i=0,1, \ldots, n-1$.

The above inclusion is strict. Consider the following example for this.

Example 3.1. We consider the lacunary sequence $\theta=\left(2^{r}\right)$ and the sequence of uncertain variables to be $\left(\xi_{k}\right)=\left(k_{n-1}\right)$. Then $\triangle_{m}^{n}\left(\xi_{k}\right)=0, \triangle_{m}^{n} x_{k}=\sum_{v=0}^{n-1}(-1)^{v}\left(\begin{array}{c}n-1 \\ v\end{array}\right) x_{k+m v}$, for all $k \in$ $\mathbb{N}$. Therefore $\left(\xi_{k}\right) \in\left[N_{\theta}^{U}, \triangle_{m}^{n}\right]_{0}$ but $\left(\xi_{k}\right) \notin\left[N_{\theta}^{U}, \triangle_{m}^{n-1}\right]_{0}$.

Theorem 3.4. The spaces of uncertain sequences $\left[N_{\theta}^{U}, \triangle_{m}^{n}\right]_{Z}, \quad Z=0,1, \infty$ are not monotone. 
To show the spaces are not monotone we consider the following example.

Example 3.2. We prove for $\left[N_{\theta}^{U}, \triangle_{m}^{n}\right]_{0}$. For $m=n=2$, consider the lacunary sequence $\theta=\left(2^{r}\right)$ and the uncertainty space $(\Gamma, \mathcal{L}, \mathcal{M})$ to be $\left\{\gamma_{1}, \gamma_{2}, \ldots\right\}$ with the power set and for any event $\Lambda \in \mathcal{L}$,

$$
\mathcal{M}\{\Lambda\}=\sum_{\gamma_{k} \in \Lambda} \frac{1}{2^{k}}
$$

Define the uncertain variables by

$$
\xi_{k}\left(\gamma_{j}\right)=\left\{\begin{array}{l}
2^{k}, \quad \text { if } j=k \\
0, \quad \text { otherwise }
\end{array}\right.
$$

Then $\left\{\xi_{k}\right\} \in\left[N_{\theta}^{U}, \triangle_{m}^{n}\right]_{0}$. Consider the sequence $\left\{\eta_{k}\right\}$ in its pre-image space defined by

$$
\eta_{k}\left(\gamma_{j}\right)=\left\{\begin{array}{l}
\xi_{k}, \quad \text { if } k=i^{2} \in \mathbb{N} \\
0, \quad \text { otherwise. }
\end{array}\right.
$$

Then $\left\{\eta_{k}\right\} \notin\left[N_{\theta}^{U}, \triangle_{m}^{n}\right]_{0}$. Hence the space is not monotone. The spaces are not solid follows by Lemma 3.1.

Theorem 3.5. The spaces of uncertain sequences $\left[N_{\theta}^{U}, \triangle_{m}^{n}\right]_{Z}, Z=0,1, \infty$ are not symmetric.

For this we consider the following example.

Example 3.3. We show this for $\left[N_{\theta}^{U}, \triangle_{m}^{n}\right]_{0}$, similar examples can be constructed for the other two spaces. For $m=n=2$, consider the lacunary sequence $\theta=\left(2^{r}\right)$. Consider the uncertainty space $(\Gamma, \mathcal{L}, \mathcal{M})$ to be $\left\{\gamma_{1}, \gamma_{2}, \gamma_{3}, \ldots\right\}$ with the power set and for any event $\Lambda \in \mathcal{L}$ such that

$$
\mathcal{M}\{\Lambda\}= \begin{cases}\sup _{\gamma_{k} \in \Lambda} \frac{k}{2 k+1}, & \text { if } \sup _{\gamma_{k} \in \Lambda} \frac{k}{2 k+1}<0.5 \\ 1-\sup _{\gamma_{k} \in \Lambda^{c}} \frac{k}{2 k+1}, & \text { if } \sup _{\gamma_{k} \in \Lambda^{c}} \frac{k}{2 k+1}<0.5 \\ 0.5, & \text { otherwise. }\end{cases}
$$

Define uncertain variables by

$$
\xi_{k}\left(\gamma_{j}\right)= \begin{cases}k, & \text { if } j=k \\ 0, & \text { otherwise. }\end{cases}
$$

Then it can be verified that the sequence $\left\{\xi_{k}\right\}$ for $k \in I_{r}$ and $r=1,2,3, \ldots$ is in $\left[N_{\theta}^{U}, \triangle_{m}^{n}\right]_{0}$. Consider the sequence $\left\{\eta_{i}\right\}$ which is a rearrangement of the sequence $\left\{\xi_{k}\right\}$ defined by $\eta_{i}(\gamma)=\left\{\xi_{1}, \xi_{4}, \xi_{9}, \xi_{2}, \xi_{10}, \ldots\right\} \notin\left[N_{\theta}^{U}, \triangle_{m}^{n}\right]_{0}$. Thus $\left[N_{\theta}^{U}, \triangle_{m}^{n}\right]_{0}$ are not symmetric in general.

Next we prove some inclusion results, for our convenience we shall denote $\left[N_{\theta}^{U}, \triangle_{m}^{n}\right]_{Z}$ for $Z=0,1, \infty$ by $\left[N_{\theta}^{U}, \triangle_{m}^{n}\right]$.

Theorem 3.6. $\left|\sigma_{1}\right|^{U}\left(\triangle_{m}^{n}\right) \subset\left[N_{\theta}^{U}, \triangle_{m}^{n}\right]$ if and only if $\liminf _{r} q_{r}>1$.

Proof. Let $\liminf q_{r}>1$, then there exists $\delta>0$ such that, $1+\delta \leq q_{r}$ for all $r \geq 1$.

Then for $\xi=\left\{\xi_{i}(\gamma)\right\} \in\left|\sigma_{1}\right|^{U}\left(\triangle_{m}^{n}\right)$ we have,

$$
\begin{aligned}
\tau_{r} & =\frac{1}{h_{r}} \sum_{i=1}^{k_{r}}\left\|\triangle_{m}^{n} \xi_{i}(\gamma)\right\|-\frac{1}{h_{r}} \sum_{i=1}^{k_{r-1}}\left\|\triangle_{m}^{n} \xi_{i}(\gamma)\right\| \\
& =\frac{k_{r}}{h_{r}}\left(\frac{1}{k_{r}} \sum_{i=1}^{k_{r}}\left\|\triangle_{m}^{n} \xi_{i}(\gamma)\right\|\right)-\frac{k_{r-1}}{h_{r}}\left(\frac{1}{k_{r-1}} \sum_{i=1}^{k_{r}}\left\|\triangle_{m}^{n} \xi_{i}(\gamma)\right\|\right)
\end{aligned}
$$


Since $h_{r}=k_{r}-k_{r-1}$, we have $\frac{k_{r}}{h_{r}} \leq \frac{1+\delta}{\delta}$ and $\frac{k_{r-1}}{h_{r}} \leq \frac{1}{\delta}$.

Now, $\frac{1}{k_{r}} \sum_{i=1}^{k_{r}}\left\|\triangle_{m}^{n} \xi_{i}(\gamma)\right\|$ and $\frac{1}{k_{r-1}} \sum_{i=1}^{k_{r}}\left\|\triangle_{m}^{n} \xi_{i}(\gamma)\right\|$ both converges to 0 , that is, $\left\{\xi_{i}(\gamma)\right\} \in$ $\left[N_{\theta}^{U}, \triangle_{m}^{n}\right]$. Therefore, $\left|\sigma_{1}\right|^{U}\left(\triangle_{m}^{n}\right) \subset\left[N_{\theta}^{U}, \triangle_{m}^{n}\right]$.

Conversely suppose that $\liminf _{r} q_{r}=1$. Since $\theta$ is lacunary, we can obtain a subsequence $\left\{k_{r_{j}}\right\}$ of $\theta$ satisfying, $\frac{k_{r_{j}}^{r}}{k_{r_{j}-1}}<1+\frac{1}{j}$ and $\frac{k_{r_{j}-1}}{k_{r_{j-1}}}>j$, where $r_{j} \geq r_{j-1}+2$. Let $\zeta(\gamma)$ and $\eta(\gamma)$ be two distinct uncertain variables. Define $\xi=\left\{\xi_{k}(\gamma)\right\}$ by

$$
\triangle_{m}^{n} \xi_{i}(\gamma)= \begin{cases}\zeta, & \text { if } i \in I_{r_{j}} \text { for some } j \in \mathbb{N} \\ \eta, & \text { otherwise. }\end{cases}
$$

Then for any uncertain variable $\rho(\gamma)$,

$$
\frac{1}{h_{r_{j}}} \sum_{I_{r_{j}}}\left\|\triangle_{m}^{n} \xi_{i}(\gamma)-\zeta(\gamma)\right\|=\|\zeta(\gamma)-\rho(\gamma)\| ; j=1,2, \ldots
$$

and

$$
\frac{1}{h_{r}} \sum_{I_{r}}\left\|\triangle_{m}^{n} \xi_{i}(\gamma)-\eta(\gamma)\right\|=\|\eta(\gamma)-\rho(\gamma)\| \quad \text { for } \quad r \neq r_{j} .
$$

It follows that $\left\{\xi_{i}(\gamma)\right\} \notin\left[N_{\theta}^{U}, \triangle_{m}^{n}\right]$. Consider $t$ is sufficiently large then there exists an unique $j$ for which $k_{r_{j}-1}<t \leq k_{r_{j+1}-1}$ and write

$$
\frac{1}{t} \sum_{i=1}^{t}\left\|\triangle_{m}^{n} \xi_{i}(\gamma)\right\| \leq \frac{k_{r_{j-1}}+h_{r_{j}}}{k_{r_{j}}-1} \leq \frac{1}{j}+\frac{1}{j}=\frac{2}{j}
$$

Now, if $t \rightarrow \infty$, it follows that $j \rightarrow \infty$. Hence $\left\{\xi_{i}(\gamma)\right\} \in\left|\sigma_{1}\right|^{U}\left(\triangle_{m}^{n}\right)$. Thus $\left\{\xi_{i}(\gamma)\right\}$ is strongly summable.

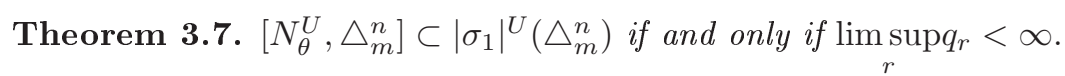

Proof. Let limsup $q_{r}<\infty$, there exists $H>0$ such that $q_{r}<H$ for all $r \geq 1$. Considering $\xi=\left\{\dot{\xi}_{i}(\gamma)\right\} \in\left[N_{\theta}^{U}, \triangle_{m}^{n}\right]$ and $\varepsilon>0$ we can find $R>0$ and $K>0$ such that $\sup \tau_{i}<\varepsilon, \tau_{i}<K$ for all $i=1,2, \ldots$ Then if $t$ is any integer with $k_{r-1}<t \leq k_{r}$, where $i \geq R$

$r>R$, and that $t$ is any integer with $k_{r-1}<t \leq k_{r}$, then we can write

$$
\begin{aligned}
\frac{1}{t} \sum_{i=1}^{t}\left\|\triangle_{m}^{n} \xi_{i}(\gamma)\right\| \leq & \frac{1}{k_{r-1}} \sum_{i=1}^{t}\left\|\triangle_{m}^{n} \xi_{i}(\gamma)\right\| \\
= & \frac{1}{k_{r-1}}\left(\sum_{I_{1}}\left\|\triangle_{m}^{n} \xi_{i}(\gamma)\right\|+\ldots+\sum_{I_{r}}\left\|\triangle_{m}^{n} \xi_{i}(\gamma)\right\|\right) \\
= & \frac{1}{k_{r-1}} \tau_{1}+\frac{k_{2}-k_{1}}{k_{r-1}} \tau_{2}+\ldots+\frac{k_{R}-k_{R-1}}{k_{r-1}} \tau_{r} \\
& \quad+\frac{k_{R+}-k_{R}}{k_{r-1}} \tau_{R+1}+\ldots+\frac{k_{r}-k_{r-1}}{k_{r-1}} \tau_{r} \\
\leq & \left(\sup _{i \geq 1} \tau_{i}\right) \frac{k_{R}}{k_{r-1}}+\left(\sup _{i \geq R} \tau_{i}\right) \frac{k_{r}-k_{R}}{k_{r-1}} \\
& =K \cdot \frac{k_{R}}{k_{r-1}}+\varepsilon . H
\end{aligned}
$$

Since $k_{r-1} \rightarrow \infty$ as $t \rightarrow \infty$, it follows that $\frac{1}{t} \sum_{i=1}^{t}\left\|\triangle_{m}^{n} \xi_{i}(\gamma)\right\| \rightarrow 0$. i.e., $\xi=\left\{\xi_{i}(\gamma)\right\} \in$ $\left|\sigma_{1}\right|^{U}\left(\triangle_{m}^{n}\right)$. 
Suppose $\lim \sup q_{r}=\infty$. In order to prove the result we need to find a sequence $\xi=$ $\left\{\xi_{i}(\gamma)\right\}$ of uncertain variables such that $\xi \in\left[N_{\theta}^{U}, \triangle_{m}^{n}\right]$ and $\xi \notin\left|\sigma_{1}\right|^{U}\left(\triangle_{m}^{n}\right)$. We select a subsequence $k_{r_{j}}$ of lacunary $\theta$ such that $q_{r_{j}}>j$. Let $\zeta(\gamma)$ and $\eta(\gamma)$ be two distinct uncertain variables and then define $\xi=\left\{\xi_{i}(\gamma)\right\}$ by

$$
\xi_{i}(\gamma)= \begin{cases}\zeta, & \text { if } k_{r_{j-1}}<i \leq 2 k_{r_{j}-1}, \text { for some } j \in \mathbb{N} \\ \eta, & \text { otherwise }\end{cases}
$$

Then

$$
\tau_{r_{j}}=\frac{1}{h_{r_{j}}} \sum_{I_{r_{j}}}\left\|\triangle_{m}^{n} \xi_{i}(\gamma)-\eta(\gamma)\right\|=\|\zeta(\gamma)-\eta(\gamma)\| \cdot \frac{k_{r_{j}-1}}{k_{r_{j}}-k_{r_{j-1}}}<\frac{1}{j-1}
$$

and $\tau_{r}=0$ if $r \neq r_{j}$. Hence, $\lim _{r} \frac{1}{h_{r}} \sum_{k \in I_{r}}\left\|\triangle_{m}^{n} \xi_{i}(\gamma)\right\|=0$. Thus $\left\{\xi_{i}(\gamma)\right\} \in\left[N_{\theta}^{U}, \triangle_{m}^{n}\right]$.

Now for the sequence $\left\{\xi_{i}\right\}$ above and for an uncertain variable $\rho$,

$$
\begin{aligned}
\frac{1}{k_{r_{j}}} \sum_{i=1}^{k_{r_{j}}}\left\|\triangle_{m}^{n} \xi_{i}(\gamma)-\rho(\gamma)\right\| \geq \frac{1}{k_{r_{j}}}\left(\sum_{i=k_{r_{j}-1}}^{2 k_{r_{j}}-1}\|\zeta(\gamma)-\rho(\gamma)\|+\sum_{i=2 k_{r_{j}-1}}^{2 k_{r_{j}}}\|\eta(\gamma)-\rho(\gamma)\|\right) \\
\geq\|\zeta(\gamma)-\rho(\gamma)\| \cdot \frac{k_{r_{j}-1}}{k_{r_{j}}}+\|\eta(\gamma)-\rho(\gamma)\| \frac{k_{r_{j}}-2 k_{r_{j}-1}}{k_{r_{j}}} \\
\geq\|\zeta(\gamma)-\rho(\gamma)\| \cdot \frac{k_{r_{j}-1}}{k_{r_{j}}}+\|\eta(\gamma)-\rho(\gamma)\| \cdot\left(1-\frac{2}{j}\right) \rightarrow\|\eta(\gamma)-\rho(\gamma)\|
\end{aligned}
$$

and

$$
\frac{1}{2 k_{r_{j}}-1} \sum_{i=1}^{2 k_{r_{j}}-1}\left\|\triangle_{m}^{n} \xi_{i}(\gamma)-\rho(\gamma)\right\| \geq \frac{k_{r_{j}-1}}{2 k_{r_{j}-1}}\|\eta(\gamma)-\rho(\gamma)\| \rightarrow \frac{\|\eta(\gamma)-\rho(\gamma)\|}{2}
$$

Consequently for any uncertain variable $\rho$, we have

$$
\begin{aligned}
\lim _{j \rightarrow \infty} \frac{1}{k_{r_{j}}} \sum_{i=1}^{k_{r_{j}}}\left\|\triangle_{m}^{n} \xi_{i}(\gamma)-\rho(\gamma)\right\|=\|\zeta(\gamma)-\rho(\gamma)\| & \neq \frac{\|\eta(\gamma)-\rho(\gamma)\|}{2} \\
& =\lim _{j \rightarrow \infty} \frac{1}{2 k_{r_{j}-1}} \sum_{i=1}^{2 k_{r_{j}-1}}\left\|\triangle_{m}^{n} \xi_{i}(\gamma)-\rho(\gamma)\right\| .
\end{aligned}
$$

Hence $\left\{\xi_{i}(\gamma)\right\} \notin\left|\sigma_{1}\right|^{U}\left(\triangle_{m}^{n}\right)$.

The following result is the consequence of the above two theorems.

Theorem 3.8. $\left[N_{\theta}^{U}, \triangle_{m}^{n}\right]=\left|\sigma_{1}\right|^{U}\left(\triangle_{m}^{n}\right)$ if and only if $1<\liminf _{r} q_{r}<\infty$.

Theorem 3.9. $|A C|^{U}\left(\triangle_{m}^{n}\right) \subset\left[N_{\theta}^{U}, \triangle_{m}^{n}\right]$.

Proof. Let $\left\{\xi_{i}(\gamma)\right\} \in|A C|^{U}\left(\triangle_{m}^{n}\right)$ and $\varepsilon>0$, there exists $N>0$ and $L(\gamma)$ such that

$$
\frac{1}{h_{r}} \sum_{i \in I_{r}}\left\|\triangle_{m}^{n} \xi_{i+n}(\gamma)-L(\gamma)\right\|<\varepsilon \quad \text { for } \quad n>N, \quad r=1,2,3, \ldots
$$

Since $\theta$ is lacunary we can choose $R>0$ such that $r \geq R$ implies $h_{r}>N$ and consequently $\tau_{r}<\varepsilon$. Thus $\left\{\xi_{i}(\gamma)\right\} \in N_{\theta}$. Thus to obtain a sequence in $N_{\theta}$ but not in $|A C|$ define $\xi=\left\{\xi_{i}(\gamma)\right\}$ by

$$
\xi_{i}(\gamma)= \begin{cases}1, & \text { if for some } r, \quad k_{r-1}<i \leq k_{r-1}+\sqrt{h_{r}} \\ 0, & \text { otherwise }\end{cases}
$$


Therefore, $\xi$ contains arbitrarily long strings of 0's and 1's, from which it follows that $\xi$ is not strongly almost convergent.

But, $\tau_{r}=\frac{1}{h_{r}} \sum_{I_{r}}\left\|\xi_{i}\right\|=\frac{1}{h_{r}}\left[\sqrt{h_{r}}\right]=\frac{1}{\sqrt{h_{r}}}$ which converges to 0 as $r \rightarrow \infty$.

\section{Generalized Difference Lacunary Convergence of Complex Uncertain Sequences with Respect to Mean, Measure,Almost Surely}

In this section we define the lacunary convergence concepts of difference uncertain sequences and derive the relations between them.

Definition 4.1. The complex uncertain sequence $\left\{\xi_{k}\right\}$ is said to be lacunary strongly convergent almost surely to $L$ with respect to difference sequence if for every $\varepsilon>0$ there exists an event $\Lambda$ with $\mathcal{M}\{\Lambda\}=1$ such that

$$
\lim _{r \rightarrow \infty} \frac{1}{h_{r}} \sum_{k \in I_{r}}\left\|\triangle_{m}^{n} \xi_{k}(\gamma)-L(\gamma)\right\|=0
$$

for every $\gamma \in \Lambda$.

Definition 4.2. The complex uncertain sequence $\left\{\xi_{k}\right\}$ is said to be lacunary strongly convergent in measure to $L$ with respect to difference sequence if

$$
\lim _{r \rightarrow \infty} \mathcal{M}\left[\left\{\gamma \in \Gamma: \frac{1}{h_{r}} \sum_{k \in I_{r}}\left\|\triangle_{m}^{n} \xi_{k}(\gamma)-L(\gamma)\right\|>\varepsilon\right\}\right]=0,
$$

for every $\varepsilon>0$.

Definition 4.3. The complex uncertain sequence $\left\{\xi_{k}\right\}$ is said to be lacunary strongly convergent in mean to $L$ with respect to difference sequence if

$$
\lim _{r \rightarrow \infty} E\left[\frac{1}{h_{r}} \sum_{k \in I_{r}}\left\|\triangle_{m}^{n} \xi_{k}(\gamma)-L(\gamma)\right\|\right]=0
$$

for every $\varepsilon>0$.

Definition 4.4. Let $\Phi_{1}, \Phi_{2}, \Phi_{3}, \ldots$ be the complex uncertainty distributions of complex uncertain variables $\xi_{1}, \xi_{2}, \xi_{3}, \ldots$, respectively. We say the complex uncertain sequence $\left\{\xi_{k}\right\}$ lacunary strong convergent in distribution to $L$ with respect to difference sequence if for every $\varepsilon>0$,

$$
\lim _{r \rightarrow \infty} \frac{1}{h_{r}} \sum_{k \in I_{r}}\left\|\triangle_{m}^{n} \Phi_{k}(c)-\Phi(c)\right\|=0
$$

for all complex $c$ at which $\Phi(c)$ is continuous.

Definition 4.5. The complex uncertain sequence $\left\{\xi_{n}\right\}$ is said to be convergent uniformly almost surely to $L$ if there exists an sequence of events $\left\{E_{k}\right\}, \mathcal{M}\left\{E_{k}\right\} \rightarrow 0$ such that $\left\{\xi_{n}\right\}$ converges uniformly to $L$ in $\Gamma-E_{k}$, for any fixed $k \in \mathbb{N}$.

The relations among the convergence concepts of complex uncertain sequences are discussed below.

Theorem 4.1. If the complex uncertain sequence $\left\{\xi_{k}\right\}$ lacunary strongly convergent in mean to $L$ with respect to difference sequence, then $\left\{\xi_{k}\right\}$ lacunary strongly converges in measure to $L$. 
Proof. It follows from the Markov's inequality that for any given $\varepsilon>0$, we have

$$
\begin{aligned}
\lim _{r \rightarrow \infty} \mathcal{M}\left[\left\{\gamma \in \Gamma: \frac{1}{h_{r}} \sum_{k \in I_{r}}\left\|\triangle_{m}^{n} \xi_{k}(\gamma)-L(\gamma)\right\|>\varepsilon\right\}\right] \\
\leq \lim _{r \rightarrow \infty} \frac{E\left[\frac{1}{h_{r}} \sum_{k \in I_{r}}\left\|\triangle_{m}^{n} \xi_{k}(\gamma)-L(\gamma)\right\|\right]}{\varepsilon} \rightarrow 0
\end{aligned}
$$

as $k \in I_{r}$. Thus $\left\{\xi_{k}\right\}$ lacunary strongly converges in measure to $L$ with respect to difference sequence and the theorem is thus proved.

But the converse of the above theorem is not true in general. i.e. lacunary strong convergence in measure with respect to difference sequence does not imply lacunary strong convergence in mean with respect to difference sequence always. This can be illustrated from the example below.

Consider the uncertainty space $(\Gamma, \mathcal{L}, \mathcal{M})$ to be $\left\{\gamma_{1}, \gamma_{2}, \ldots\right\}$ with power set and

$$
\mathcal{M}\{\Lambda\}= \begin{cases}\sup _{\gamma_{i} \in \Lambda} \frac{1}{i}, & \text { if } \sup _{\gamma_{i} \in \Lambda} \frac{1}{i}<0.5 \\ 1-\sup _{\gamma_{i} \in \Lambda^{c}} \frac{1}{i}, & \text { if } \sup _{\gamma_{i} \in \Lambda^{c}} \frac{1}{i}<0.5 \\ 0.5, & \text { otherwise. }\end{cases}
$$

and the complex uncertain variables be defined by

$$
\xi_{i}\left(\gamma_{j}\right)= \begin{cases}i, & \text { if } j=i \\ 0, & \text { otherwise }\end{cases}
$$

for $i \in I_{r}$ and $L \equiv 0$. For $\varepsilon>0$, we have

$$
\begin{aligned}
\lim _{r \rightarrow \infty} \mathcal{M} & \left(\left\{\gamma \in \Gamma: \frac{1}{h_{r}} \sum_{k \in I_{r}}\left\|\triangle_{m}^{n} \xi_{i}(\gamma)-L(\gamma)\right\|>\varepsilon\right\}\right) \\
& =\lim _{r \rightarrow \infty} \mathcal{M}\left(\left\{\gamma \in \Gamma: \frac{1}{h_{r}} \sum_{k \in I_{r}}\left\|\triangle_{m}^{n} \xi_{i}(\gamma)\right\|>\varepsilon\right\}\right) \\
& =\lim _{r \rightarrow \infty} \mathcal{M}\left(\left\{\gamma_{i}\right\}\right) \\
& =\lim _{r \rightarrow \infty} \frac{1}{i} \rightarrow 0 \quad\left(\text { as } \quad i \in I_{r} .\right)
\end{aligned}
$$

The sequence $\left\{\xi_{i}\right\}$ lacunary strongly converges in measure to $L$. However for each $i \in I_{r}$, we have the uncertainty distribution of uncertain variable $\left\|\xi_{i}-L\right\|=\left\|\xi_{i}\right\|$ is

$$
\begin{aligned}
& \Phi_{i}(x)= \begin{cases}0, & \text { if } x<0 ; \\
1-\frac{1}{i}, & \text { if } 0 \leq x<i ; \\
1, & \text { otherwise. }\end{cases} \\
& E\left[\frac{1}{h_{r}} \sum_{k \in I_{r}}\left\|\triangle_{m}^{n} \xi_{k}(\gamma)-L(\gamma)\right\|\right]=\int_{0}^{+\infty} \mathcal{M}\{\xi \geq x\} d x-\int_{-\infty}^{0} \mathcal{M}\{\xi \leq x\} d x \\
&=\int_{0}^{i} 1-\left(1-\frac{1}{i}\right) d x \\
&=1
\end{aligned}
$$

That is, the $\left\{\xi_{i}(\gamma)\right\}$ does not converge in mean to $L(\gamma)$ with respect to difference sequence. 
Lemma 4.1. Let the complex uncertain sequence $\left\{\xi_{n}\right\}$ has real part $\left\{\zeta_{n}\right\}$ and imaginary part $\left\{\eta_{n}\right\}$, respectively, for $n=1,2, \ldots$. If uncertain sequences $\left\{\zeta_{n}\right\}$ and $\left\{\eta_{n}\right\}$ converge in measure to $L_{1}$ and $L_{2}$ respectively, then the complex uncertain sequence $\left\{\xi_{n}\right\}$ converge in measure to $L=L_{1}+i L_{2}$.

Theorem 4.2. Let the complex uncertain sequence $\left\{\xi_{k}\right\}$ has real part $\left\{\zeta_{k}\right\}$ and imaginary $\left\{\eta_{k}\right\}$, respectively, for $k \in I_{r}$. If uncertain sequences $\left\{\zeta_{k}\right\}$ and $\left\{\eta_{k}\right\}$ lacunary strongly convergent in measure to $L_{1}$ and $L_{2}$ respectively with respect to difference operators on sequence, then complex uncertain sequence $\left\{\xi_{k}\right\}$ lacunary strongly uniformly convergent with respect to the difference operator in distribution to $L=L_{1}+i L_{2}$.

Proof. Let $c=a+i b$ be a point at which the complex uncertainty distribution $\Phi$ is continuous. For any $\alpha>a, \beta>b$, we have,

$$
\begin{gathered}
\left\{\zeta_{k} \leq a, \eta_{k} \leq b\right\}=\left\{\zeta_{k} \leq a, \eta_{k} \leq b, \mathrm{E}_{1} \leq \alpha, \mathrm{E}_{2} \leq \beta\right\} \cup\left\{\zeta_{k} \leq a, \eta_{k} \leq b, \mathrm{E}_{1}>\alpha, \mathrm{E}_{2}>\beta\right\} \\
\cup\left\{\zeta_{k} \leq a, \eta_{k} \leq b, \mathrm{E}_{1} \leq \alpha, \mathrm{E}_{2}>\beta\right\} \cup\left\{\zeta_{k} \leq a, \eta_{k} \leq b, \mathrm{E}_{1}>\alpha, \mathrm{E}_{2} \leq \beta\right\} \\
\subset\left\{\mathrm{E}_{1} \leq \alpha, \mathrm{E}_{2} \leq \beta\right\} \cup\left\{\left\|\triangle_{m}^{n} \zeta_{k}(\gamma)-\mathrm{E}_{1}(\gamma)\right\| \geq \alpha-a\right\} \cup\left\{\left\|\triangle_{m}^{n} \eta_{k}(\gamma)-\mathrm{E}_{2}(\gamma)\right\| \geq \beta-b\right\} .
\end{gathered}
$$

It follows from the subadditivity axiom that

$$
\begin{aligned}
\Phi_{k}(c)=\Phi_{k}(a+i b) \leq \Phi(\alpha+i \beta)+\mathcal{M}\{\gamma & \left.\in \Gamma:\left\|\triangle_{m}^{n} \zeta_{k}(\gamma)-L_{1}(\gamma)\right\| \geq \alpha-a\right\} \\
& +\mathcal{M}\left\{\gamma \in \Gamma: \| \triangle_{m}^{n} \eta_{k}(\gamma)-L_{2}(\gamma) \mid \geq \beta-b\right\} .
\end{aligned}
$$

Since $\left\{\zeta_{k}\right\}$ and $\left\{\eta_{k}\right\}$ lacunary strongly convergent in measure to $L_{1}$ and $L_{2}$ respectively. So for $\varepsilon>0$ and $k \in I_{r}$ we have,

$$
\begin{aligned}
& \lim _{r \rightarrow \infty} \mathcal{M}\left\{\gamma \in \Gamma: \frac{1}{h_{r}} \sum_{k \in I_{r}}\left\|\triangle_{m}^{n} \zeta_{k}(\gamma)-L_{1}(\gamma)\right\| \geq(\alpha-a) \geq \varepsilon\right\}=0, \\
& \lim _{r \rightarrow \infty} \mathcal{M}\left\{\gamma \in \Gamma: \frac{1}{h_{r}} \sum_{k \in I_{r}}\left\|\triangle_{m}^{n} \eta_{k}(\gamma)-L_{2}(\gamma)\right\| \geq(\beta-b) \geq \varepsilon\right\}=0 .
\end{aligned}
$$

Thus we have, $\limsup \Phi_{k}(c) \leq \Phi(\alpha+i \beta)$ for any $\alpha>a, \beta>b$. Letting $\alpha+i \beta \rightarrow a+i b$, we get,

$$
\limsup _{r \rightarrow \infty} \Phi_{k}(c) \leq \Phi(c)
$$

On the other hand, for any $x<a, y<b$ we have,

$$
\begin{gathered}
\left\{L_{1} \leq x, L_{2} \leq y\right\}=\left\{\zeta_{k} \leq a, \eta_{k} \leq b, L_{1} \leq x, L_{2} \leq y\right\} \cup\left\{\zeta_{k} \leq a, \eta_{k} \leq b, L_{2} \leq x, L_{2} \leq y\right\} \\
\cup\left\{\zeta_{k}>a, \eta_{k} \leq b, L_{1} \leq x, L_{2} \leq y\right\} \cup\left\{\zeta_{k}>a, \eta_{k}>b, L_{1} \leq x, L_{2} \leq y\right\} \\
\subset\left\{\zeta_{k} \leq a, \eta_{k} \leq b\right\} \cup\left\{\left\|\triangle_{m}^{n} \zeta_{k}-L_{1}\right\| \geq a-x\right\} \cup\left\{\left\|\triangle_{m}^{n} \eta_{k}-L_{2}\right\| \geq b-y\right\} .
\end{gathered}
$$

which implies

$$
\begin{aligned}
\Phi(x+i y) \leq \Phi_{k}(a+i b)+\mathcal{M}\left\{\gamma \in \Gamma:\left\|\triangle_{m}^{n} \zeta_{k}(\gamma)-L_{1}(\gamma)\right\| \geq a-x\right\} \\
+\mathcal{M}\left\{\gamma \in \Gamma:\left\|\triangle_{m}^{n} \eta_{k}(\gamma)-L_{2}(\gamma)\right\| \geq b-y\right\} .
\end{aligned}
$$

Since

$$
\begin{aligned}
& \lim _{r \rightarrow \infty} \mathcal{M}\left\{\gamma \in \Gamma: \frac{1}{h_{r}} \sum_{k \in I_{r}}\left(\left\|\triangle_{m}^{n} \zeta_{k}(\gamma)-L_{1}(\gamma)\right\| \geq a-x\right) \geq \varepsilon\right\}=0, \\
& \lim _{r \rightarrow \infty} \mathcal{M}\left\{\gamma \in \Gamma: \frac{1}{h_{r}} \sum_{k \in I_{r}}\left(\left\|\triangle_{m}^{n} \eta_{k}(\gamma)-L_{2}(\gamma)\right\| \geq b-y\right) \geq \varepsilon\right\}=0,
\end{aligned}
$$


we obtain

$$
\Phi(x+i y) \leq \lim \inf _{r \rightarrow \infty} \Phi_{k}(a+i b)
$$

for any $x<a, y<b$. Taking $x+i y \rightarrow a+i b$, we get

$$
\Phi(c) \leq \liminf _{r \rightarrow \infty} \Phi_{k}(c) .
$$

It follows from (4.1) and (4.2) that $\Phi_{k}(c) \rightarrow \Phi(c)$ as $r \rightarrow \infty$ and $k \in I_{r}$. That is the complex uncertain sequence $\left\{\xi_{k}\right\}$ is lacunary strongly convergent in distribution to $L=L_{1}+i L_{2}$.

Converse of the above theorem is not necessarily true. i.e. Lacunary strongly convergent in distribution with respect to difference sequence does not imply lacunary strongly convergence in measure with respect to that difference sequence. Following example illustrates this.

Consider the uncertainty space $(\Gamma, \mathcal{L}, \mathcal{M})$ to be $\left\{\gamma_{1}, \gamma_{2}\right\}$ with $\mathcal{M}\left\{\gamma_{1}\right\}=\mathcal{M}\left\{\gamma_{2}\right\}=\frac{1}{2}$. We define a complex uncertain variable as

$$
\xi(\gamma)= \begin{cases}1, & \text { if } \gamma=\gamma_{1} \\ -1, & \text { if } \gamma=\gamma_{2}\end{cases}
$$

We also define $\left\{\xi_{k}\right\}=-\xi$, for $k \in I_{r}$. Then $\left\{\xi_{k}\right\}$ and $\xi$ have the same distribution and thus $\left\{\xi_{k}\right\}$ converges in distribution to $\xi$. However, for any given $\varepsilon>0$, we have

$$
\begin{array}{r}
\lim _{r \rightarrow \infty} \mathcal{M}\left[\left\{\gamma \in \Gamma: \frac{1}{h_{r}} \sum_{k \in I_{r}}\left\|\triangle_{m}^{n} \xi_{k}(\gamma)-\xi(\gamma)\right\|>\varepsilon\right\}\right] \\
\quad=\lim _{r \rightarrow \infty} \mathcal{M}\left[\left\{\gamma \in \Gamma: \frac{1}{h_{r}} \sum_{k \in I_{r}}\left\|\triangle_{m}^{n} 2 \xi_{k}(\gamma)\right\|>\varepsilon\right\}\right] \neq 0
\end{array}
$$

Therefore, the sequence $\left\{\xi_{k}\right\}$ does not lacunary strongly converge in measure to $\xi$ with respect to difference sequence.

Theorem 4.3. Let $\xi_{1}, \xi_{2}, \xi_{3}, \ldots$ be complex uncertain variables. Then $\left\{\xi_{k}\right\}$ is lacunary strongly convergent almost surely to $L$ with respect to difference sequence if and only if for any $\varepsilon>0$, we have,

$$
\mathcal{M}\left(\bigcap_{r \in I_{r_{k}}} \bigcup_{k \in I_{r}}\left\{\gamma \in \Gamma: \frac{1}{h_{r}} \sum_{k \in I_{r}}\left\|\triangle_{m}^{n} \xi_{k}(\gamma)-L(\gamma)\right\|>\varepsilon\right\}\right)=0
$$

Proof. By the definition of lacunary strongly convergent almost surely we have that there exists an event $\Lambda$ with $\mathcal{M}(\Lambda)=1$, such that

$$
\lim _{r \rightarrow \infty} \frac{1}{h_{r}} \sum_{k \in I_{r}}\left\|\triangle_{m}^{n} \xi_{k}(\gamma)-L(\gamma)\right\|=0
$$

for every $\gamma \in \Lambda$.

Then for any $\varepsilon>0$, there exists $m$ such that $\frac{1}{h_{r}} \sum_{k \in I_{r}}\left\|\triangle_{m}^{n} \xi_{k}(\gamma)-L(\gamma)\right\|<\varepsilon$, where $k>m$, for any $\gamma \in \Lambda$, which is equivalent to

$$
\mathcal{M}\left(\bigcup_{r \in I_{r_{k}}} \bigcap_{k \in I_{r}}\left\{\gamma \in \Gamma: \frac{1}{h_{r}} \sum_{k \in I_{r}}\left\|\triangle_{m}^{n} \xi_{k}(\gamma)-L(\gamma)\right\|>\varepsilon\right\}\right)=1 .
$$

But using the duality axiom it follows that

$$
\mathcal{M}\left(\bigcap_{r \in I_{r_{k}}} \bigcup_{k \in I_{r}}\left\{\gamma \in \Gamma: \frac{1}{h_{r}} \sum_{k \in I_{r}}\left\|\triangle_{m}^{n} \xi_{k}(\gamma)-L(\gamma)\right\|>\varepsilon\right\}\right)=0 .
$$


Hence the result is proved.

Theorem 4.4. Let $\xi_{1}, \xi_{2}, \xi_{3}, \ldots$ be complex uncertain variables. If $\left\{\xi_{k}\right\}$ is lacunary strongly convergent uniformly almost surely to $L$ with respect to difference sequence, then $\left\{\xi_{k}\right\}$ is lacunary strongly convergent in measure to $L$ with respect to that difference sequence.

Proof. If $\left\{\xi_{k}\right\}$ is lacunary strongly convergent uniformly almost surely to $L$ with respect to difference sequence then

$$
\lim _{r \rightarrow \infty} \mathcal{M}\left(\bigcup_{k \in I_{r}}\left\{\gamma \in \Gamma: \frac{1}{h_{r}} \sum_{k \in I_{r}}\left\|\triangle_{m}^{n} \xi_{k}(\gamma)-L(\gamma)\right\|>\varepsilon\right\}\right)=0
$$

from the above theorem.

But

$$
\begin{aligned}
& \mathcal{M}\left(\left\{\gamma \in \Gamma: \frac{1}{h_{r}} \sum_{k \in I_{r}}\left\|\triangle_{m}^{n} \xi_{k}(\gamma)-L(\gamma)\right\|>\varepsilon\right\}\right) \\
& \quad \leq \mathcal{M}\left(\bigcup_{k \in I_{r}}\left\{\gamma \in \Gamma: \frac{1}{h_{r}} \sum_{k \in I_{r}}\left\|\triangle_{m}^{n} \xi_{k}(\gamma)-L(\gamma)\right\|>\varepsilon\right\}\right)
\end{aligned}
$$

Therefore, $\left\{\xi_{k}\right\}$ is lacunary strongly convergent in measure to $L$ with respect to difference operator on sequence.

\section{REFERENCES}

[1] A. Esi, B. C. Tripathy and B. Sarma, On some new type generalized difference sequence spaces, Mathematica Slovaca, 57(2007), no. 5, 475-482.

[2] A. R. Freedman, J. J. Sember and M. Raphael, Some Cesaro-type summability spaces, Proc London Math Soc., 37(1978), no. 3, 508-520.

[3] B. C. Tripathy and S. Mahanta, On a class of sequences related to the $\ell^{p}$ space defined by Orlicz functions, Soochow journal of Mathematics, 29(2003), no. 4, 379-391.

[4] B. C. Tripathy and S. Mahanta, On a class of generalized lacunary difference sequence spaces defined by Orlicz functions, Acta Mathematicae Applicatea Sinica, English Series,20(2004),231-238.

[5] B. C. Tripathy and A. Esi, A new type of difference sequence spaces, Internat. J. Sci.Tech.,1(2006), $11-14$.

[6] B. C. Tripathy and P. Nath, Statistical Convergence of Complex Uncertain Sequences, New Math. Nat. Comput., 13(2017), no. 4, 359-374.

[7] B. C. Tripathy and P. J. Dowari, Nörlund and Riesz mean of sequence of complex uncertain variables, Filomat, 32(2018), no. 8, 2875-2881.

[8] B. Liu, Some Research Problems in Uncertainty Theory, Journal of Uncertain Systems, 3(2009), no. $1,3-10$.

[9] B. Liu, Uncertainity Theory, 5th Ed., Springer-Verlag, Berlin, (2016).

[10] C. You, On the Convergence of Uncertain Sequences, Mathematical and Computer Modelling, 49(2009), no. (3-4), 482-487.

[11] G. G. Lorentz, A contribution to the theory of divergent sequences, Acta Math. 80(1948),167-190.

[12] H. Kizmaz, On certain sequence spaces, Canadian Math. Bull. 24(1981 ), 169-176.

[13] J. P. King, Almost summable sequences, Proc. Amer. Math. Soc., 16(1966), 1219-1225.

[14] M. Et and R. Colak, On some generalized difference sequence spaces, Soochow J. Math., 21(1995), no. 4, 377-386.

[15] Ö. Kisi, Lacunary $I_{\sigma}$-Statistical Convergence of Complex Uncertain Sequence, Sigma Journal of Engineering and Natural Sciences, 37(2019), no. 2, 507-520.

[16] Ö. Kisi,, $S_{\lambda}(I)$ - Convergence of Complex Uncertain Sequence, Matematychni Studii, 51(2019), no. 2, 183-194.

[17] P. J. Dowari and B. C. Tripathy, Lacunary convergence of sequences of complex uncertain variables, Bol. Soc. Paran. Mat.,(Accepted for publication).

[18] P. K. Kamthan and M. Gupta, Sequence spaces and Series, Lecture Notes in Pure and Applied Mathematics, 65. Marcel Dekker, Inc., New York, (1981). 
[19] X. Chen, Y. Ning and X. Wang, Convergence of complex uncertain sequences, J. Intell. Fuzzy Syst., 30(2016), no. 6, 3357-3366 .

Pranab Jyoti Dowari: pranabdowari@gmail.com

Department of Mathematics, Tripura University, Suryamaninagar, Agartala-799022, Tripura India

Binod Chandra Tripathy: tripathybc@yahoo.com; tripathybc@rediffmail.com

Department of Mathematics, Tripura University, Suryamaninagar, Agartala-799022, Tripura India

Received 17/02/1919; Revised 07/11/2020 